\title{
Editorial for the Topical Issue: Recent Breakthroughs in Microplasma Science and Technology ${ }^{\star}$
}

\author{
Kurt H. Becker ${ }^{\mathrm{a}}$ \\ Department of Applied Physics and Department of Mechanical and Aerospace Engineering, New York University Tandon School \\ of Engineering, Brooklyn, NY 11201, New York, USA
}

Received 12 March 2016

Published online 3 May 2016 - (c) EDP Sciences, Società Italiana di Fisica, Springer-Verlag 2016

\begin{abstract}
Microplasmas are plasmas where at least one dimension is in the submillimeter range. They can be thermal plasmas, where the gas temperature is far above the room temperature and is approaching the electron temperature, or they can be based on glow discharges and thus non-thermal in nature, with gas temperatures much below electron temperatures. The attraction of non-thermal microplasmas is that they can be operated stably at high gas pressures, in rare gases as well as in molecular gases and in gas mixtures, excited by direct current (dc) as well as by pulsed $\mathrm{dc}$ and alternate current (ac), radio frequency (rf), or microwaves. This allows one to exercise unprecedented control of the plasma operating parameters over a wide range and also makes these plasmas a versatile tool for many applications.
\end{abstract}

Research into the basic properties of microplasmas gained prominence and recognition as a well-defined and stand-alone area within the larger field of plasma science and technology about 20 years ago. Since then, the activity in microplasma research and applications has continuously increased. A survey of peer reviewed papers on microplasmas published annually shows an order of magnitude increase in the number of papers published from 1995 to 2015 and this excludes papers that deal exclusively with technological applications where the microplasma is used solely as a tool. This Topical Issue aims to provide a snap shot of the current state of microplasma research and applications.

This issue contains a comprehensive Topical Review entitled "20 years of microplasma research: a status report" which focuses primarily on the status of microplasma science and our understanding of the physics principles that enable microplasma operation. Where appropriate, microplasma applications are also addressed. However, the discussion of microplasma applications in this review is limited to examples where the application is closely tied to the plasma science. No attempt is made in this Topical Review to provide a comprehensive and in-depth review of the diverse range of all microplasma applications, except for the inclusion of a few key references to recent reviews of microplasma applications. Other articles in this Topical Issue address structure formation in atmospheric-pressure discharges, gas breakdown and plasma impedance in split-ring resonators, results of a study of biomolecule and polymer surface modifications by a surface discharge, a study of discharge processes and electric modeling of an atmospheric-pressure plasma jet in argon, the spatial stabilization of dielectric barrier discharge microfilaments by residual heat build-up in air, and other topics. Areas of application of microplasmas such as environmental remediation, biological decontamination, UV light sources, microdischarge-based sensors, photonic devices, guided ionization waves in plasma jets, and microwave-sustained microplasmas are beyond the scope of this Topical Issue. Likewise, the rapidly expanding area of plasma medicine, incl. dental applications of microplasmas is not covered here Applications of microplasmas will be discussed in detail in a forthcoming issue of the European Physical Journal Special Topics.

\footnotetext{
* Contribution to the Topical Issue "Recent Breakthroughs in Microplasma Science and Technology", edited by Kurt Becker, Jose Lopez, David Staack, Klaus-Dieter Weltmann and Wei Dong Zhu.

${ }^{a}$ e-mail: kurt.becker@nyu.edu
} 